\title{
Ultrasound-assisted catheter-directed low-dose thrombolysis for acute pulmonary embolism - A case report
}

\author{
Fibrinólisis de baja dosis facilitada por ultrasonido dirigida por catéter en \\ tromboembolismo pulmonar agudo. Reporte de un caso
}

\author{
Héctor H. Escutia-Cuevas ${ }^{1 *}$, Marco A. Alcántara-Meléndez ${ }^{1}$, Paulette M. Fuentes-Espinosa ${ }^{1}$, \\ Guillermo Orozco-Guerra ${ }^{1}$, Álvaro D. Rentería-Valencia', Ronald E. Rivas-Gálvez², \\ Manuel A. Espinoza-Rueda², Robinsson J. Vásquez-Orozco², and Hiram Vela-Vizcaíno²
}

${ }^{1}$ Department of Interventional Cardiology; ${ }^{2}$ Cardiology Division, Centro Médico Nacional 20 de Noviembre. Instituto de Seguridad y Servicios Soẹiales de los Trabajadores del Estado (ISSSTE), Mexico City, Mexico

Pulmonary thromboembolism (PTE) is one of the main causes of morbidity and mortality throughout the world. The annual incidence of PTE is approximately 600,000 cases, and there are 100,000-180,000 related deaths, which makes it the main preventable cause of death among hospitalized patients'. The 30-day mortality rate in patients with PTE who develop cardiogenic shock ranges from $16 \%$ to $25 \%$ and in patients who suffer cardiac arrest from $52 \%$ to $65 \%^{2}$.

As for clinical classification, a division can be established between massive or high-risk and submassive or intermediate-risk PTE. Regarding massive pulmonary embolism, it is characterized by hemodynamic instability and is a condition with high mortality associated, up to 3 times higher in comparison with patients who remain hemodynamically stable. This condition is currently defined by the presence of hypotension with systolic blood pressure (SBP) $<90 \mathrm{mmHg}$ for more than 15 min or needs for inotropic support to maintain SBP above $90 \mathrm{mmHg}$. Submassive or intermediate-risk pulmonary embolism is defined by SBP $>90 \mathrm{mmHg}$ with evidence of the right heart failure (dilated right ventricle
[RV] and an RV to left ventricle [LV] ratio $>0.9$ in the four-chamber view on tomography or echocardiogram) and elevated biomarkers, such as troponin or natriuretic peptide B. Patients are at high risk of disease progression if they are not treated in a timely manner ${ }^{3}$.

Computed tomography (CT) pulmonary angiography allows not only proper visualization of PTE up to the segmental level but also visualization of the LV assan indicator of dysfunction. Echocardiography is an acceptable diagnostic alternative, although generalfy it does not provide a definitive diagnosis and neither does it excludes PTE, but it can confirm or rule out overfoad and severe RV dysfunction?.

At present, stratification scales in the form of scores, such as the pulmonary embolism severity index (PESI), the Wells score, or the Geneva score, are of great help, along with hemodynamic and imaging findings, to classify the patient according to his/her mortality risk ${ }^{3}$.

The optimal treatment strategy continues to be a reason for controversy since PTE mortality is comparable to that of acute myocardial infarction, without achieving a mortality reduction having been possible

\section{Correspondence:}

*Héctor H. Escutia-Cuevas

Avenida Coyoacán 1617, Interior APH7

Col. Del Valle Sur, Del. Benito Juárez

C.P. 03104, Ciudad de México, Mexico City, México

Date of reception: 2-10-2018

Date of acceptance: 4-12-2018

E-mail: perseoyarista@ hotmail.com

DOI: 10.24875/ACME.M19000053
Available online: 06-05-2019 Arch Cardiol Mex (Eng). 2019;89(3):262-266 www.archivoscardiologia.com 2604-7063/C 2019 Instituto Nacional de Cardiología Ignacio Chávez. Published by Permanyer. This is an open access article under the CC BY-NC-ND license (http://creativecommons.org/licenses/by-nc-nd/4.0/). 
over the past three decades ${ }^{4}$. For patients with hemodynamic instability secondary to massive pulmonary embolism, the treatment of choice continues to be systemic fibrinolysis ${ }^{5}$. Over the past few years, new evidence has emerged indicating that, both in massive and submassive embolism, catheter-guided and ultrasound-assisted low-dose thrombolysis is a highly effective treatment that reduces RV overload and decreases pulmonary pressure and thrombus burden, without increasing the risk of hemorrhage when added to the treatment with heparin ${ }^{6-8}$.

Catheter-directed therapy (CDT) can be used as an alternative to thrombolysis when a patient has absolute contraindications to systemic fibrinolysis, or if he/she is at very high risk of major bleeding, as adjunctive therapy when thrombolysis has failed to improve hemodynamics, or as an alternative to surgery if immediate access is not available. The goal of CDT is to eliminate thrombotic obstructions of the main pulmonary arteries to facilitate RV mechanics and thus improve survival. The clinical success rate, defined as stabilization of hemodynamic parameters, resolution of hypoxia, and survival until discharge, was $87 \%{ }^{9}$.

Experience in our country with this treatment modality is still limited, with few previous reports existing. For this reason, and in an effort to continue generating experience on this subject, herein, we describe a clinical case that exemplifies the ideal use of CDT in a patient with submassive PTE.

\section{Case report}

A 78-year-old female patient, with a history of long-standing hypertension, as well as an ischemic stroke 2 years prior, without apparent sequels was admitted to the emergency department of our center with due to a 6 min episode of sudden dyspnea at rest, accompanied by oppressive chest pain, diaphoresis, and nausea. Biochemically, she had serum troponin I elevation $(130 \mathrm{pg} / \mathrm{mL})$ and, electrocardiographically, she had depression of the ST segment on the lower wall. On physical examination, she was found with sleepiness, blood pressure at $95 / 60 \mathrm{mmHg}$, heart rate $130 \mathrm{bpm}$, respiratory rate $26 \mathrm{bpm}$, with $82 \%$ saturation on pulse oximetry (without ventilatory support), Grade III jugular ingurgitation, symmetric chest without stridor or wheezing, and limbs without edema. Transthoracic echocardiogram: LV with $43 \mathrm{~mL}$ end-diastolic volume, $21 \mathrm{~mL}$ end-systolic volume, $70 \%$ ejection fraction, without alterations in global or segmental mobility at rest; left atrial volume of $30 \mathrm{~mL} / \mathrm{m}^{2}$; mitral valve without regurgitation, tricuspid aortic valve, without significant transvalvular gradient, without insufficiency; dilated RV, with severely depressed systolic function, $12 \mathrm{~mm}$ tricuspid annular plane systolic excursion (TAPSE), $5 \mathrm{~cm} / \mathrm{s}$ S-wave, 15\% fraction shortening; dilated right atrium without thrombi within; structurally normal tricuspid valve, with coaptation deficit generating concentric jet of moderate insufficiency, $5 \mathrm{~mm}$ vena contracta, $75 \mathrm{mmHg}$ regurgitation gradient; pulmonary artery-systolic pressure (PASP) was calculated to be $85 \mathrm{~mm} H \mathrm{Hg}$ with a 0.98 RV/LV ratio (Fig. 1A). Pulmonary angiography showed RV dilation, RV/LV ratio of 0.99 , as well as filling defect with total distal occlusion in the main branch of the right pulmonary artery (Fig. 1B and C), which confirms the diagnosis of acute submassive. PTE (Class V PESI score, of very high risk, with 10-24\% 30-day mortality associated).

Anticoagulation with unfractionated heparin was started $(80 \mathrm{lU} / \mathrm{kg}$ intravenous bolus and then continuous infusion until quickly reaching and maintaining the prolongation of activated partial thromboplastin time between 1.5 and 2.5 times the control), and due to the existence of contraindications, not performing thrombolysis was decided. With the purpose to assess the CDT, the emergency right heart catheterization was performed, with pulmonary angiography and measufing the pressures of the right cavities, with the following being found: PASP $90 \mathrm{mmHg}$, mean pulmonary artery pressure (MPAP) $50 \mathrm{mmHg}$, with slow flow and cloudy image in the right main branch, with the absence of distal flow in the right lower pulmonary branch (Fig.2A), and pulmonary valve without pullback gradient tracing toward RV outflow tract.

During the same procedure, an endovascular system EKOS probe (BTG-IM, Bothell, Washington) was placed as follows: through a French size 6 venous introducer, a 0.035 hydrophilic guide was advanced in the fight femoral vein, through which a multipurpose $5 \mathrm{Fr}$ cürve catheter was advanced to perform measurements of the previously commented cavities. Subsequently the aforementioned pulmonary angiography was performed. Based on the findings, the hydrophilic gutide was advanced toward the right branch, and then, tot the right lower lobar artery; an exchange was made for the EKOS probe and was placed from the distal segment to the bifurcation of the pulmonary trunk (Fig. 1D): adequate operation of the console and adequate infusion of the drug and coolant were corroborated, withothe procedure being concluded without complications

Due to the high risk of bleeding, starting alteplase continuous infusion at $1 \mathrm{mg} / \mathrm{h}$ during $12 \mathrm{~h}$ was decided. 

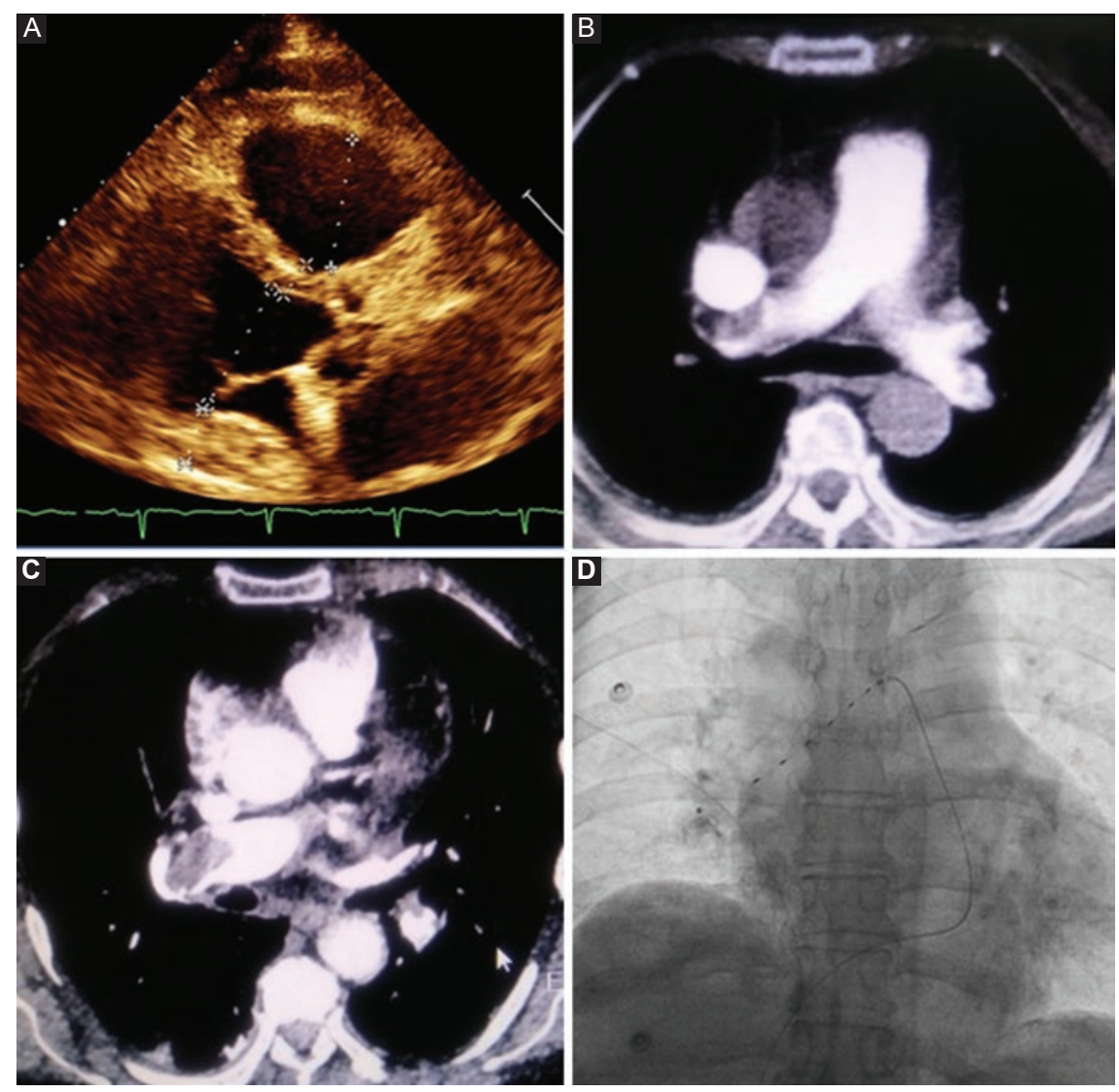

Figure 1. Pulmonary thromboembolism diagnostic phase and treatment. A: initial transthoracic echocardiogram, parasternal long axis, where the right ventricle (RV) dilation is observed, RV/left ventricle ratio of 0.98 . B: pulmonary computed tomography angiography axial slice, with filling defect in the right branch of the pulmonary artery that extends to subsegmental branches. C: axial slice at the T5 level with higher definition of the right pulmonary branch filling defect. D: final fluoroscopic result with EKOS probe (BTG-IM, Bothell, Washington) placed from the distal segment of the right lower lobar artery to the bifurcation of the pulmonary trunk.

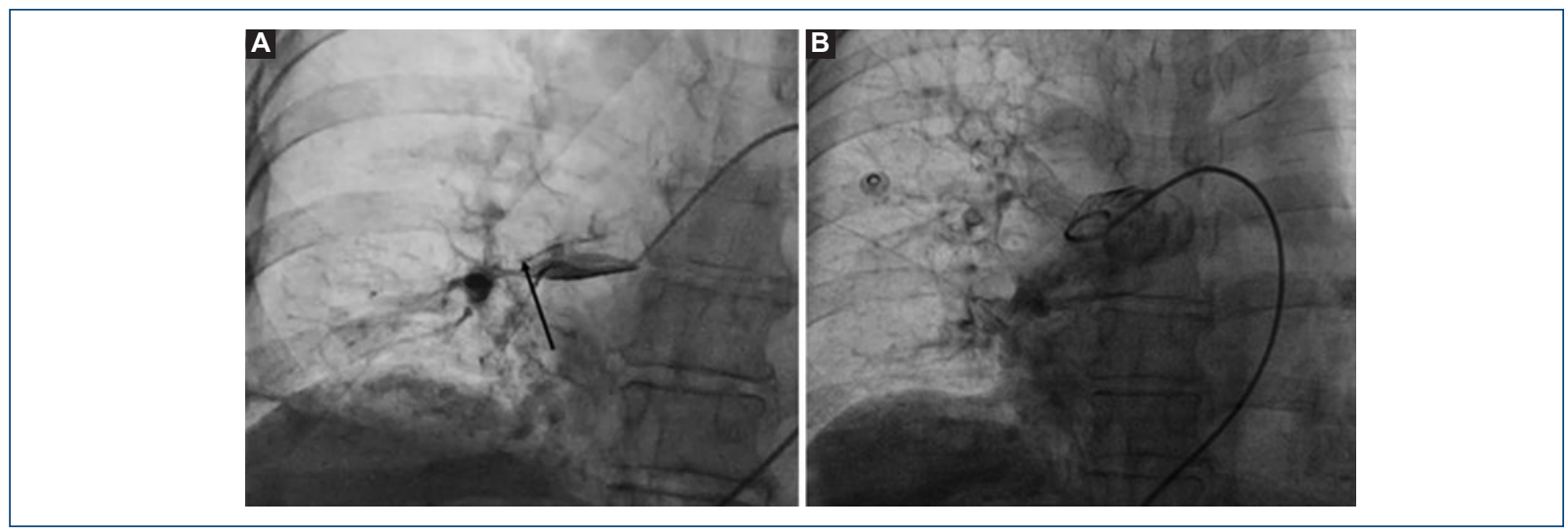

Figure 2. Pulmonary angiography before and after treatment. A: in anteroposterior projection, a hazy image (arrowa) is observed in the right main branch with no distal flow in both subsegmental branches. B: post-procedure pulmonary angiography, which shows distal flow improvement in the subsegmental branches and disappearance of the image suggestive of thrombus in the right main branch. 
Subsequently, the EKOS probe was removed, with evident hemodynamic improvement. Echocardiographically: RV with preserved systolic function, TAPSE 19, S-wave of $10 \mathrm{~cm} / \mathrm{s}, 45 \%$ shortening fraction; tricuspid valve with coaptation deficit that generated concentric jet of moderate insufficiency, $50 \mathrm{mmHg}$ PASP, and $\mathrm{RV} / \mathrm{LV}$ ratio of 0.78 . Control angiography and pressure measurement $48 \mathrm{~h}$ after the CDT showed a $48 \mathrm{mmHg}$ PASP, $30 \mathrm{mmHg}$ MPAP, and normal distal flow in the right branch of the pulmonary artery, without images suggestive of thrombus (Fig. 2B).

The patient continued hospitalized on hemodynamics observation for $72 \mathrm{~h}$ and was then discharged asymptomatic with the New York Heart Association functional Class I for outpatient follow-up on anticoagulation based on rivaroxaban $20 \mathrm{mg} /$ day. After 2 months' follow-up, she continued asymptomatic from the cardiovascular point of view, with no new embolism events.

\section{Discussion}

In patients without an absolute contraindication for systemic thrombolysis, CDT can be considered, as it was in this clinical case. Low-energy ultrasound disaggregates fibrin inside acute thrombi. This is taken advantage of by the EKOS device (BTG-IM, Bothell, Washington), which combines low-energy ultrasound emission and infusion of a thrombolytic agent through a catheter that contains multiple side orifices. This strategy was assessed in the ULTIMA ${ }^{7}$ trial, which demonstrated superiority to anticoagulation alone for improving hemodynamics without a significant increase in hemorrhagic complications. The SEATTLE II study was a single group CDT multicenter trial that demonstrated an improvement in RV hemodynamic indices in patients with massive and submassive PTE ${ }^{6}$. There were no cases of intracranial hemorrhage, although 16 bleeding episodes ( 1 serious GUSTO and 15 moderate GUSTO) were observed.

Given the available evidence on CDT in acute PTE, the use of an ultrasound-assisted CDT strategy should be explored in a highly individualized form. As shown in this case, patients with confirmed acute PTE must be quickly anticoagulated with unfractionated intravenous heparin with an $80 \mathrm{lU} / \mathrm{kg}$ bolus followed by continuous infusion ${ }^{10}$. Together with patient clinical and hemodynamic evaluation, individualized decision should be made with regard to his/her suitability for CDT. Continuous infusion of alteplase at $1 \mathrm{mg} / \mathrm{h}$ into each pulmonary artery can be started while the patient is being observed in an intensive care setting ${ }^{8}$. The alteplase dose can be reduced by half at $5 \mathrm{~h}$ for an additional $10 \mathrm{~h}$ if patient bleeding risk requires it ${ }^{10} \mathrm{Al}-$ teplase maximum recommended dose is 20 mgo for bilateral placement of the device, and at $15 \mathrm{~h}$, at most, the alteplase infusion should be discontinued along with the ultrasound transducer system ${ }^{10}$. The catheter and introducer sheath system should be removed with manual compression of the site of access until hemostasis is achieved. A follow-up echocardiogram should be performed to confirm the improvement in theo RV function.

This case is presented as an example of a realiand descriptive clinical approach to the use of this treatment modality for acute PTE. It is a case with adequate ical and hemodynamic evolution in the short term, and procedures previously suggested in the existing literature are, therefore, reproduced.

CDT appears to be a viable and safe option, with $10 \mathrm{w}$ risk of complications, which should be individually used in cases of patients with massive or submassive PTE with contraindications for thrombolytic treatment or at high risk of hemorrhage.

\section{Conflicts of interest}

The authors declare that they have no conflicts of interest.

\section{Funding}

None.

\section{Ethical disclosures}

Protection of people and animal subjects. The authors declare that no experiments were performed on humans or animals for this study.

Confidentiality of data. The authors declare that they have followed the protocols of their work center on the publication of patient data.

Right to privacy and informed consent. The authors have obtained written informed consent of the patients and/or subjects mentioned in the article. The corresponding author is in possession of this document.

\section{References}

1. Stephen R, Ken Z, Noor A. Acute Pulmonary Embolism: Endovascular Therapy. Cardiovasc Diagn Ther. 2018; 8(3):244-52.

2. Yamamoto T. Management of Patients with High-Risk Pulmonary Embolism: A Narrative Review. J Intens Care. 2018;6:16. 
3. Jimenez $D$, Yusen RD, Otero $R$, Uresandi $F$, Nauffal $D$, Laserna $E$. Prognostic Models for Selecting Patients with Acute Pulmonary Embolism for Initial Outpatient Therapy. Chest. 2007;132(1):24-30.

4. Kuo WT, Van den Bosch MA, Hofmann LV, Louie JD, Kothary N, Sze DY. Catheter-directed embolectomy, fragmentation, and thrombolysis for the treatment of massive pulmonary embolism after failure of systemic thrombolysis. Chest. 2008;134:250-4.

5. Konstantinides SV, Barco S, Lankeit M, Meyer G. Management of Pulmonary Embolism an Update. J Am Coll Cardiol. 2016;67(8):976-90.

6. Piazza G, Hohlfelder B, Jaff MR, Ouriel K, Engelhardt TC, Sterling KM, et al. A Prospective, Single-Arm, Multicenter Trial of Ultrasound-Facilitated, Catheter-Directed, Low-Dose Fibrinolysis for Acute Massive and Submassive Pulmonary Embolism: The SEATTLE II Study. JACC Cardiovasc Interv. 2015;8(10):1382-92.
7. Kucher $N$, Boekstegers $P$, Müller $O$, Kupatt $C$, Beyer-Westendorf J Heitzer T, el al. Randomized, Controlled Trial of Ultrasound-Assisted Catheter-Directed Thrombolysis for Acute Intermediate-Risk Pulmonary Embolism. Circulation. 2013:129:479-86.

8. Tapson VF, Sterling K, Jones N, Elder M, Tripathy U, Brower J, et al. A Randomized Trial of the Optimum Duration of Acoustic Pulse Thrombolysis Procedure in Acute Intermediate-Risk Pulmonary Embolism: The OPTALYSE PE Trial. JACC Cardiovasc Interv. 2018;11(14):1401-10.

9. Bloomer TL, El-Hayek GE, McDaniel MC, Sandvall BC, Liberman HA, Devireddy CM, et al. Safety of Catheter-Directed Thrombolysis for Massive and Submassive Pulmonary Embolism: Results of a Multicenter Registry and Meta-Analysis. Cath Cardiovasc Interv. 2017;89(4):754-60.

10. Jaber WA, Fong PP, Weisz G, Lattouf O, Jenkins J, Rosenfield K et al. Acute Pulmonary Embolism: With an Emphasis on an Interventional Approach. J Am Coll Cardiol. 2016;67(8):991-1002. 